\title{
LÉGITIMATION ET COMMUNICATION
}

\author{
Elisabeth Volckrick
}

Les métiers de la communication se sont beaucoup développés et professionnalisés au cours des dernières décennies. La plupart d'entre eux s'exercent dans des organisations privées, publiques et associatives. Ces organisations communiquent tant en interne qu'en externe afin d'informer, échanger, se faire connaître, donner leur opinion, gérer leur image, dénoncer, se justifier et se défendre parfois. Chacune de ces organisations, de façon différente, produit et gère des contenus culturels qui ont tous une fonction de légitimation par rapport aux différents acteurs avec lesquels elle est en contact.

Au terme de cette recherche, il est apparu que les professionnels de la communication d' organisation se retrouvent plongés au cœur de logiques d'actions fort différentes, voire même contradictoires : entre les objec-

1 Elisabeth Volckrick est professeur invité au Département de communication de l’Université catholique de Louvain (marie-elisabeth.volckrick@uclouvain.be).

Recherches en communication, $\mathrm{n}^{\circ} 25$ (2006). 
tifs de la direction et les objectifs particuliers, entre le social et l'économique, entre les contraintes internes et les contraintes externes, entre des exigences d'efficacité et de justesse, etc. Comment vont-ils s'y prendre pour préserver la pluralité des intérêts et des positions des acteurs ? Quels mondes de référence, quel type d'argumentation, chacun mobilisera-t-il dans son action? Que nous révèlent les pratiques professionnelles des métiers de la communication quant à la nature des processus de légitimation mis en cuvre par les organisations? Telle est la question qui sous-tend les contributions rassemblées dans cette revue. Notre ambition est de donner un premier aperçu de cette mise en œuvre des processus de légitimation par les organisations, en combinant des analyses conceptuelles, des élaborations théoriques et des études de pratiques concrètes et actuelles de métiers de la communication d'organisation.

Comme toute publication, celle-ci représente pour une part un aboutissement, en l'occurrence les traces du séminaire «Métiers de la communication d'organisation et processus de légitimation » auquel prirent part les membres du Laboratoire d'analyse des systèmes de communication d'organisation (LASCO) d'octobre 2001 à juin 2006. Dés sa création en février 2000, le LASCO met en évidence des problématiques particulières : l'observation et l'analyse des métiers et pratiques professionnelles de la communication d'organisation; l'analyse des phénomènes de légitimation des organisations, des acteurs porteurs de ces processus et des formes de communication les accompagnant.

Durant les années académiques 2001-2003, un premier séminaire scientifique est mis sur pied et animé par Pierre de Saint-Georges avec l'aide de John Cultiaux. Les séances du séminaire recouvrent différentes interventions portant, notamment, sur les métiers et fonctions de la communication d'organisation (A. Gryspeerdt, Fl. Carion), sur la notion de légitimité et son application à l'analyse des organisations (G.Liénard), sur la nouvelle configuration idéologique du management et les épreuves du travail flexible (Th. Périlleux) ainsi que des réflexions de professionnels d'organisation sur leur métier ( $\mathrm{Ph}$. Massart, M. Joannes, J-M. Pierlot) ${ }^{1}$.

1 Ces différentes interventions firent l'objet d'une première publication : P. de SaintGeorges et J. Cultiaux, Métiers de la communication et processus de légitimation, Séminaire scientifique du LASCO 2001-2003, Les Cahiers du LASCO, n ${ }^{\circ} 1$, Louvain-la-Neuve, 2004. 
S'appuyant sur le construit théorique ayant émergé de ces échanges tout en l'ouvrant à la question de la normativité (A. Berten), le séminaire se poursuit, durant les années académiques 2003-2006, sous la responsabilité d'Elisabeth Volckrick avec l'aide de Stéphanie Rigo. La démarche se centre sur le rôle de «constructeur de consensus » du professionnel de la communication d'organisation. La question de la légitimité étant souvent soulevée lors d'une situation conflictuelle, les membres du séminaire se sont intéressés aux «épreuves » spécifiques de cette activité professionnelle, aux conditions dans lesquelles ces acteurs (se) légitiment au travers de leurs actions et dans le cadre de leur mandat. Le choix méthodologique ${ }^{1}$ fut pris de travailler à partir d'expériences concrètes relatées et analysées par des professionnels de la communication avec les chercheurs du Lasco. Trois modules de travail se sont constitués : un module médiation institutionnelle, un module socio-humanitaire et un module entreprise publique mixte. Sur ces trois modules se sont greffés progressivement d'autres travaux (Th. Libaert, J-M. Pierlot) et des recherches doctorales (O. Le Saëc, L. A. Blason).

Chacun des textes rassemblés dans ce volume, tout en étant sous la seule responsabilité de son auteur, s'inscrit de façon plus ou moins forte dans ce projet collectif commun. Dans la partie « repères », les deux premiers articles ont pour objectif de mettre en perspective les multiples pratiques professionnelles des métiers de la communication d'organisation. La délimitation même de ces métiers s'avère problématique. Les professionnels, qui apparaissent généralement comme des «éclaireurs », semblent paradoxalement assez silencieux sur leurs pratiques qui sont souvent méconnues par le grand public. Axel Gryspeerdt et Florence Carion établissent ainsi un relevé des métiers sous forme d'un inventaire contextualisé et ébauchent un premier rangement en cinq grandes catégories : généraliste en communication, spécialiste interface médias, éditorialiste d'organisation, médiateur, lobbyiste. Ces métiers ont, en tout cas, en commun d'être des activités où des personnes ont reçu un mandat pour agir dans le domaine de la communication et d'être aux prises avec des questions de légitimation : la leur et celle de leur organisation. Tenter un classement des métiers ne peut faire oublier que le champ de la communication est lui-même en constante et rapide évolution.

1 Nous nous sommes inspirés de la démarche d'analyse en groupe. 
Poursuivant cette exploration, Thierry Libaert s'intéresse plus spécialement aux tendances actuelles de la communication d'entreprise et nous montre qu'elle traverse aujourd'hui une période de transition. La communication, l'entreprise et la communication d'entreprise ont été remis en cause. Le thème de la confiance est central dans la plupart des communications d'entreprise : «c'est la parole même de l'entreprise qui est en débat ». Quant au communicateur, sa situation est paradoxale : on exige de lui qu'il informe toujours plus, mieux et vite, et, dans le même temps, on lui signifie que sa parole n'est pas crédible. Les opérations de communication se veulent de plus en plus interactives, directes et de proximité avec les publics et les exigences de rentabilité immédiates. La légitimité de l'entreprise tend à devenir une question centrale à mesure que s'amplifie la demande actuelle de responsabilité sociale et environnementale. Cet élargissement à d'autres champs que l'économie et la diversité des discours et intérêts qui en résultent posent problème : la potentialité des conflits s'accroît. Il apparaît nécessaire à l'entreprise de trouver les moyens de créer du consensus entre les acteurs et d'éviter que les conflits n'entravent son développement.

Un constat semble s'imposer : la légitimité des organisations, de leurs dirigeants, de leurs communications et des communicateurs tend à devenir une question insistante. Les conflits récents entre pouvoirs publics, ONG, opinion publique, médias, entre actionnaires et administrateurs, entre administrés et administration, entre partenaires sociaux, le rappellent. Le concept même de légitimation est actuellement confronté à des défis importants. Pour certains, une reformulation complexe de la grammaire du lien social est en voie d'émergence dans nos sociétés contemporaines et a anticipé une mutation des régimes pratiques de gouvernance et de légitimation. A bien des égards, les processus de légitimation impliquent désormais la participation des individus et donc devient légitime ce à quoi vont participer les individus qui produisent la légitimité.

Deux approches conceptuelles se distinguent ici, elles traversent les différentes contributions et témoignent d'une dialectique bien présente dans nos travaux et nos échanges. Selon une première approche, le concept de légitimation vise à décrire un processus (et ses résultats) par lequel des individus sont amenés à reconnaître la légitimité du pouvoir, des institutions, des comportements, des usages, des conventions, des 
discours, etc., C'est ainsi que Georges Liénard examine, dans sa contribution, les concepts de légitimité, de légitimation, de pouvoir et de domination symbolique. Il montre combien les processus de légitimation et de légitimité sont l'ossature des rapports de pouvoir ou de domination symbolique. En appliquant son cadre théorique et analytique aux relations de pouvoir ou de domination symbolique qui s'exerce sur les métiers de la communication dans les organisations, il fait apparaitre un enjeu central : les métiers de la communication agissent sur la légitimité ou l'illégitimité de l'ordre social. L'article de Pierre de Saint-Georges fait écho à cet enjeu lorsqu'il évoque le " communicateur-symptôme », révélateur en quelque sorte de l'objet et du système de légitimation mis en place. Le métier de communicateur est inscrit dans une sorte de paradoxe de base : devoir dévoiler (faire connaître, éclairer le projet organisationnel) et occulter en même temps (ne pas donner à voir le rapport de forces social sous-jacent à ce qu'on doit faire connaître).

Selon une seconde approche, le concept de légitimation doit être abordé en prenant davantage en compte la dimension éthique du rapport aux normes telle qu'elle est principalement impliquée, selon Habermas, dans l'usage communicationnel du discours. Pour André Berten, la question est de savoir s'il y a ou non une justification normative à ce qui est reconnu comme légitime. Il souligne, dans sa contribution, l'importance des processus cognitifs spécifiques qui sont en jeu dans les croyances en la légitimité éthique d'un ordre social quelconque, d'un rôle ou d'une organisation. Sa théorie de la normativité permet de repenser la fonction des croyances dans une telle légitimité et de soutenir que c'est dans la communication discursive que l'on peut trouver un certain type de fondement à celle-ci.

Les contributions qui sont rassemblées dans la partie « recherches » de cet ouvrage présentent les résultats des travaux menés en commun avec des professionnels de certains métiers de la communication d'organisation.

Les pratiques des médiateurs institutionnels dans la fonction publique font l'objet de l'article d'Elisabeth Volckrick et Stéphanie Rigo. La fonction d'administrer s'est fortement modifiée au cours du temps, elle est aujourd'hui traversée par des logiques d'action extrêmement différentes, voire contradictoires. Une tension nouvelle émerge à l'intersection de l'administration et de son environnement. La question 
de la légitimité de l'action administrative se pose de façon nouvelle. La mise en place de l'institution du médiateur dans la fonction publique témoigne de l'insuffisance des mécanismes institutionnels classiques de régulation sociale et apparaît comme une tentative imaginée pour solutionner un double problème d'efficacité et de légitimité de manière systématique et régulée.

Florence Carion et John Cultiaux montrent combien les professionnels de la communication dans l'action humanitaire se situent eux aussi au centre d'un jeu d'acteurs dont les actions se justifient et se légitiment en référence à des ordres de grandeur antagonistes : marchand et civique. Ils semblent ne pas avoir les moyens, du moins actuellement, d'être des faiseurs de compromis durables : les accords qu'ils établissent sont des arrangements locaux et temporaires.

La contribution de Joseph Pirson aborde l'évolution des actions des professionnels de la communication dans les organisations semipubliques. Ses analyses révèlent un paradoxe : le modèle économique dominant exerce une pression croissante sur la définition des fonctions de la communication et, dans le même temps, l'estompement des modèles culturels traditionnels en favorise la redéfinition.

Quant à Thierry Libaert et Jean-Marie Pierlot, ils analysent, à partir de cas concrets et récents, comment pouvoirs publics, entreprises et associations construisent leur communication lors de leurs échanges ainsi que les risques et opportunités de légitimation qui en résultent pour chacune des parties. L'analyse des interactions montre que les frontières entre ces trois acteurs sont loin d'être étanches et plutôt d'une porosité croissante. Avec l'élargissement de la sphère médiatique, l'opinion publique apparaît désormais au centre des relations entre les acteurs et de ce fait retrouve un rôle central.

Olivier Le Saëc traite des relations avec la presse et plus spécialement des porte-parole de la Commission européenne. Il constate que ceux-ci s'avèrent être porte-parole des portefeuilles administratifs de la Commission européenne avant d'être ceux des acteurs politiques européens que sont les Commissaires européens. Ceci peut expliquer la prédominance d'aspects techniques et bureaucratiques dans la couverture médiatique de l'actualité communautaire européenne et être révé- 
lateur d'une logique « rationnelle-légale » prégnante dans la vie quotidienne de la Commission européenne.

Pour Lenay Alexandra Blason, les professionnels de la communication dans le secteur culturel remplissent une véritable fonction de médiation. Ils sont amenés à mettre en œuvre des moyens, des ressources et des compétences pour obtenir la légitimité de leur institution et de ses différents acteurs. Ce faisant, ils se retrouvent souvent pris en tension entre une double exigence d'efficacité et d'éthique professionnelle.

Une analyse attentive des pratiques concrètes de certains métiers de la communication dans les organisations nous montre que ces dernières connaissent des mutations importantes qui les obligent à repenser leur rapport avec leur environnement. Ces mutations s'accompagnent d'une profonde transformation des conditions de légitimation de l'ordre commun, de la norme. Le succès des métiers de la communication d'organisation en témoigne : c'est dans la communication - au sens de construction de consensus - qu'une légitimation peut désormais se produire. 